(C2006 IEEE. Personal use of this material is permitted. However, permission to reprint/republish this material for advertising or promotional purposes or for creating new collective works for resale or redistribution to servers or lists, or to reuse any copyrighted component of this work in other works must be obtained from the IEEE. 


\title{
Reputation Relationship and Its Inner Relationships for Service Oriented Environments
}

\author{
Farookh Khadeer Hussain ${ }^{1}$, Elizabeth Chang $^{1}$ and Tharam S.Dillon ${ }^{2}$ \\ ${ }^{1}$ School of Information Systems \\ Curtin University of Technology \\ P.O. Box U1987 \\ Perth, Western Australia, Australia \\ \{Farookh.Hussain, Elizabeth.Chang\}@cbs.curtin.edu.au \\ ${ }^{2}$ Faculty of Information Technology \\ University of Technology, Sydney \\ Sydney, NSW 2007, Australia \\ tharam@it.uts.edu.au
}

\begin{abstract}
Trust and Reputation are vital components for trusted e-business. In this paper, we propose a definition of trust relationship. Additionally we discuss in depth about the concept of trust relationship.

A definition for reputation relationship is proposed in this paper. From the analysis of reputation relationship we find that the reputation relationship is a composite relationship and is composed of three inner relationships.

We propose the three inner relationships and define them. We then propose the characteristics of the inner relationships with in the reputation relationship.
\end{abstract}

\section{Introduction}

The involvement of the Third Party Agents in the reputation process complicates the concept of Reputation Relationship. This is also true in the physical world. Dealing with binary relationships is much easier than dealing with ternary relationships where a Third Party is involved. In this section we discuss and elucidate with examples the interconnections between the three types of Agents involved in a reputation relationship. We additionally discuss the inner relationships with in the reputation relationship.

As we can see the intricacies and the complexities in a Reputation Relationships do not exist in a Trust Relationship. This is because the Trust Relationship is a direct binary relationship between Trusting Agent and Trusted Agent, whereas, the Reputation Relationship involves an extra Agent, the Third Party Recommendation Agent.

\section{Definition of the Reputation Relationship}

Reputation Relationship Definition: We define a Reputation Relationship in a service-oriented environment as a bond or association between the Trusting Agent, Third Party Recommenders and Trusted Agents that revolves around the reputation query. The term reputation query is very essential when defining reputation relationship because if there is no reputation query then the Trusting Agent will not be associated with the $3^{\text {rd }}$ Party Agent (in the sense of soliciting recommendations from the $3^{\text {rd }}$ party Agent) and the Trusted Agent(in the sense of soliciting recommendations about the trusted agent). Similarly the only reason why the $3^{\text {rd }}$ Party Agent is communicating recommendations about the trusted agent to the trusting agent is because of the reputation query issued by the trusting agent to it. It can be clearly seen that the following three relationships comprise the composite reputation relationship.

(a) The relationship between the Trusting Agent and the $3^{\text {rd }}$ Party Recommendation Agent, signifying the request for information about the trusted agent/ product or service by the Trusting Agent the $3^{\text {rd }}$ Party Recommendation Agent

(b) The relationship between the $3^{\text {rd }}$ Party Recommendation Agent and Trusting Agent, signifying reply to the reputation query by the $3^{\text {rd }}$ Party Recommendation Agent

(c) The relationship between the $3^{\text {rd }}$ Party Recommendation Agent and Trusting Agent.

The difference between a Reputation Relationship and a Trust Relationship is as follows: 
- There is an extra Agent known as the Third Party Agent involved in the reputation relationship which is not involved in the trust relationship.

- The Third Party Agent engages in three major activities in the reputation process; having a trust relationship with Trusted Agent, giving opinions about the Trusted Agent to the Trusting Agent, and being assessed the trusting agent for the assessment of opinion being carried out by the Trusting Agent.

- A trust relationship revolves around trust between the trusting agent and the trusted agent in a given context and time. However a reputation relationship revolves around the reputation query. If there is no reputation query then there would be no reputation relationship.

- The Third Party Agent's recommendation is the key for the trusting agent to determine the trustworthiness / QoS / QoP, of the agent / service / product.

- The correctness of the referrals communicated by the Third Party Agents, which the trusting agent may or may not know, leads to the concept of Witness trustworthiness value. The Witness Trustworthiness Value quantifies quantitatively the correctness of the recommendations communicated by a given Witness Trustworthiness Value.

In the following sections, we shall step by step reveal the inner relationships with in the Reputation Relationship.

\section{Inner Relationships within the Reputation Relationship}

The three inner relationships with in the reputation relationships are:

- Reputation Query Trust Relationship (trust relationship between Trusting Agent and Third Party Recommendation, in the context of soliciting recommendations or opinions by the Trusting Agent to the $3^{\text {rd }}$ Party Recommendation Agent)

- Recommendation Trust Relationship (trust relationship between the Third Party Recommendation Agent and the Trusting Agent, in the context of communicating recommendations by the $3^{\text {rd }}$ Party Recommendation Agent to the Trusting Agent)

- Third Party Trust Relationship (trust relationship between the Trusted Agent and the Third Party Recommendation Agent in the context and time slot specified in the reputation query).

These three inner trust relationships form the composite Reputation Relationship. The three inner relationships are known as Reputation Query Trust Relationship, Recommendation Trust Relationship and Third Party Trust Relationship.

In this section, we explained how the Third Party Agents and their involvement complicate the concept of Reputation Relationship. Additionally we mentioned the three intertwined inner relationships with in the reputation relationship due to the involvement of the $3^{\text {rd }}$ Party Agent. In the following sections, we will further explain the three inner relationships in detail.

\section{Recommendation Trust Relationship}

In this section, we will formally define and elucidate the Recommendation Trust Relationship. Additionally we enumerate the difference between the Recommendation Trust Relationship and the Trust Relationship.

Definition: The Recommendation Trust Relationship is defined as the bond or association between the Third Party Recommendation Agent and the Trusting Agent in the context of communicating recommendations at a given point in time.

The recommendation trust relationship can be regarded as an instance of a trust relationship, in which the context of the trust relationship is 'communicating recommendations', and the time is one or more time slot/s. The role of the trusting agent in the Recommendation Trust Relationship is played by the $3^{\text {rd }}$ Party Recommendation Agent in the reputation relationship and the role of the trusted agent is played by the trusting agent in the reputation relationship.

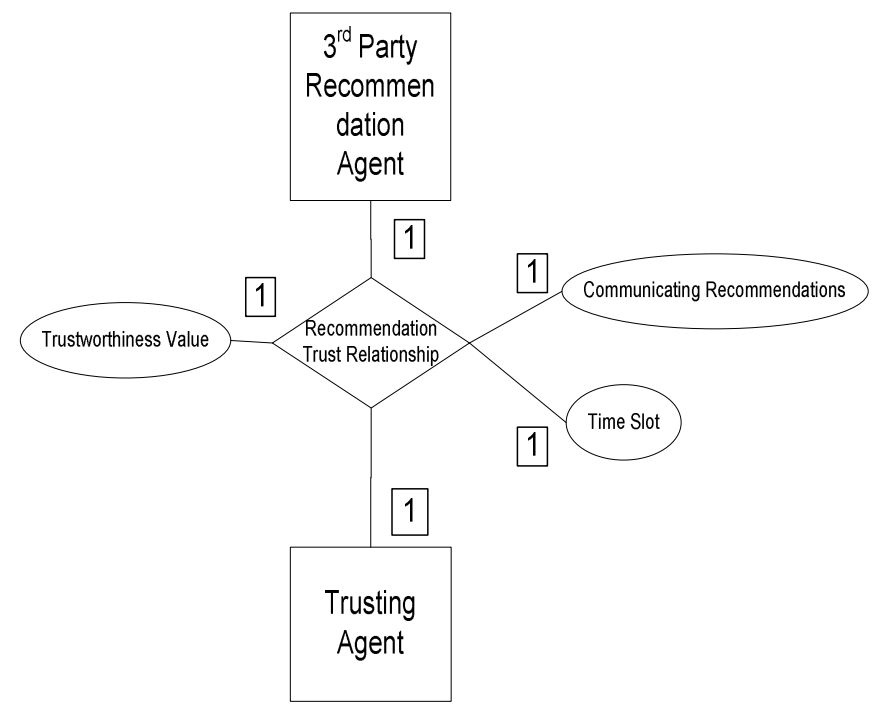

Figure 1.The Recommendation Trust Relationship Diagram

The layout of the Recommendation Trust Relationship Diagram above (Figure 1) is similar to the Trust Diagram introduced in [1]. However, the similarity is at the structural level only and not at the semantic level. As mentioned before, the role of the trusting agent in the recommendation trust relationship is played by the $3^{\text {rd }}$ Party Recommendation Agent in the reputation 
relationship. Additionally the role of the trusted agent is played by the Trusting Agent in the reputation relationship.

There is a 1:1 (One-to-One) relationship between the Third Party Recommendation Agent and the Trusting Agent in a Recommendation Trust Relationship. This means that in a given Recommendation Trust Relationship captures the relationship that a given $3^{\text {rd }}$ Party Recommendation Agent has with a single trusting agent, in the context of communicating recommendations and at a single time slot. If a given $3^{\text {rd }}$ Party Recommendation Agent has relationships with more than one trusting agent, in the context of communicating recommendations, then each of the relationships (that the $3^{\text {rd }}$ Party Recommendation Agent has) with the trusting agent is regarded as a distinct Recommendation Trust Relationship. Similarly, if a given $3^{\text {rd }}$ Party Recommendation Agent has relationships with more a single trusting agent, in the context of communicating recommendations, in more than one time slot, then each of the relationships (that the $3^{\text {rd }}$ Party Recommendation Agent has) with the trusting agent in a distinct time slot is regarded as a distinct Recommendation Trust Relationship.

\subsection{The difference between Trust Relationship and Recommendation Trust Relationship}

Despite the structural similarity of the above diagram to the Trust Model described in Chapter XX, there are fundamental differences between the Recommendation Trust Relationship diagram and the Trust Model. These are:

1) As mentioned before, a Recommendation Trust Relationship can a be regarded as an instance of trust relationship in which the role of the trusting agent and the trusted agent is played by $3^{\text {rd }}$ Party Agent and Trusting Agent of the reputation relationship respectively.

2) The context in a trust relationship could be any context. However in a Recommendation Trust Relationship, the context can only be 'communicating recommendations'.

However the trustworthiness of the Trust Relationship represents the willingness and capability of the trusted agent in a given context and at a given point in time.

The Recommendation Trust Relationship has the following additional characteristics

1) Due to the recommendation trust relationship, the $3^{\text {rd }}$ Party Recommendation Agent can communicate the repute value to the trusting agent. The Trusting Agent can then combine these repute values or opinions using some mathematical means to determine the reputation of the trusted agent.
During the process of combining the repute values or opinions from different $3^{\text {rd }}$ Party Agent using the mathematical means the trusting agent should take care that the repute values are of the same context and of the same time slot for which the trusting agent has to make a trusted based decision about the trusted agent.

2) The trusted agent of the Recommendation Trust Relationship (the trusting agent of the reputation relationship) could be Known Agents, Unknown Agents, Trustworthy Known Agents and Untrustworthy Known Agents. The $3^{\text {rd }}$ Party Recommendation Agents offer their opinions or recommendations in reply to the reputation query.

3) A given $3^{\text {rd }}$ Party Recommendation Agent may have recommendation trust relationship with more than one Trusting Agent. In other words a given $3{ }^{\text {rd }}$ Party Trust Relationship may choose to communicate recommendations to more then one trusting agent. A given $3^{\text {rd }}$ Party Recommendation Agent can reply to zeroto-many $(0: \mathrm{M})$ reputation queries.

4) If there is no recommendation query trust relationship then the recommendations by the $3^{\text {rd }}$ Party Recommendation Agents would not reach the trusting agent subsequently the reputation value for the Trusted Agent could not be generated. In other words the repute value for the trusted agent in the reputation query would not be able to reach the trusting agent in the reputation query in the absence of a recommendation trust relationship. Further more, if no $3^{\text {rd }}$ Party Recommendation Agent replies the reputation query then the trusting agent would not be able to determine the trustworthiness value / QoP / QoS, of the trusted agent / product / service.

\section{Third Party Trust Relationships}

In this section, we will formally define and elucidate the Third Party Trust Relationship. Additionally we enumerate the difference between the Third Party Trust Relationship and the Trust Relationship.

Definition: The Third Party Trust Relationship is defined as the bond or association the Third Party Recommendation Agent and the Trusted Agent in the context at a time as specified in the reputation query.

From the perspective of the Trusting Agent, who initiates the whole process of reputation computation, this relationship is between the $3^{\text {rd }}$ Party Recommendation Agent and the Trusted Agent. From the perspective of the trusting agent in the reputation relationship, the trusted agent in the reputation relationship plays the role of the 
trusted agent in the $3^{\text {rd }}$ Party trust relationship and the $3^{\text {rd }}$ Party Recommendation Agent, in reputation relationship plays the role of the trusting agent in the $3^{\text {rd }}$ Party trust relationship. Therefore, I call this relationship as Third Party Trust Relationship.

This conceptual representation of the $3^{\text {rd }}$ Party Trust Relationship is a similar to the conceptual representation of Trust Relationship proposed in [1], except that the Trusting Agent in the $3^{\text {rd }}$ Party is the is a Third Party Recommendation Agent in the reputation relationship.

Figure 2 depicts the relationship between Third Party Recommendation Agents and the Trusted Agent.

There is a 1:1 (One-to-One) relationship between the Third Party Recommendation Agent and the Trusted Agent in a $3^{\text {rd }}$ Party Trust Relationship. This means that in a given $3^{\text {rd }}$ Party Trust Relationship captures the relationship that a given $3^{\text {rd }}$ Party Recommendation Agent has with a single trusted agent, in the context and specified in the reputation query. If the reputation query comprises of enquiries about more than one trusted agent in one or more given context/s and in one or more time slot/s and if a given $3^{\text {rd }}$ Party Recommendation Agent has relationships with more than one trusted agent, specified in the reputation query, then each of the relationships (that the $3^{\text {rd }}$ Party Recommendation Agent has) with the a given trusted agent, in a unique combination of time slot and context is regarded as a distinct $3^{\text {rd }}$ Party Trust Relationship.

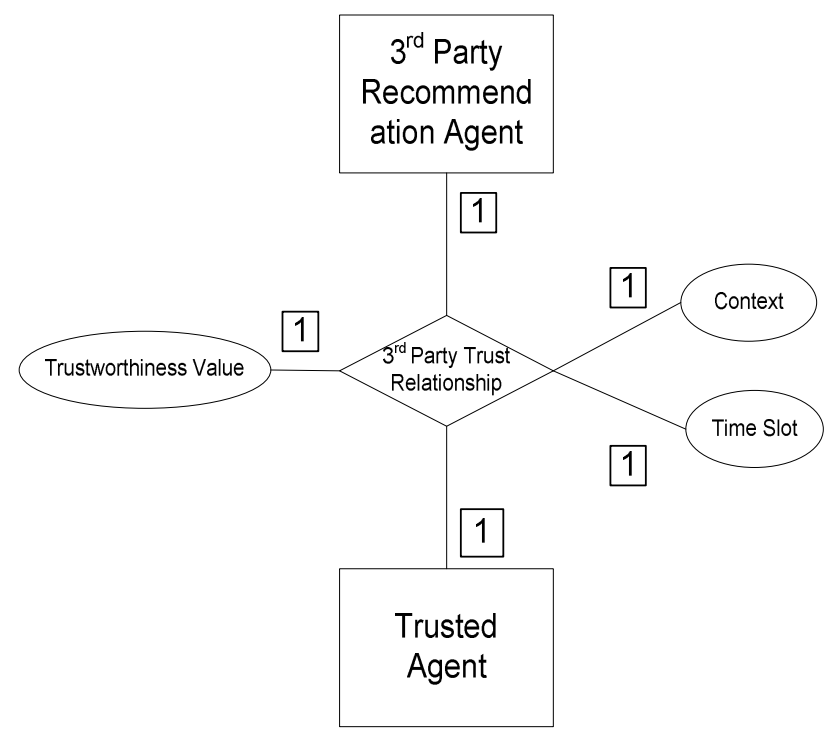

Figure 2. The Third Party Trust Relationship Diagram

\subsection{The Difference between Trust Relationship and $3^{\text {rd }}$ Party Trust Relationship}

There is a close similarity of between the conceptual representation of the Trust Model described in [1] and the conceptual representation of the $3^{\text {rd }}$ Party Trust Relationship described in Figure 2. However, there are some differences between this diagram and the Trust Model.

1) The role of the trusting agent in the $3^{\text {rd }}$ Party Recommendation Relationship is played by the $3^{\text {rd }}$ Party Recommendation Agent in the reputation relationship. Additionally the role of the trusted agent in the $3^{\text {rd }}$ Party Recommendation Relationship is played by the trusted agent in the reputation relationship.

2) The trustworthiness value assigned by the 3rd Party Recommendation Agent to the Trusted Agent in the reputation relationship is context and time dependent. When this trustworthiness value is communicated by the $3^{\text {rd }}$ Party Recommendation Agent to the other agent (Trusting Agent) it becomes the repute value.

3) The context in the Third Party trust relationship should be the same as the context in reputation query. In contrast, trust relationship between a trusting agent and a trusted agent could exist in any context.

In other words, trustworthiness assigned by the Trusting Agent to the Trusted Agent becomes the recommendation or opinion of the Trusted Agent, when the Trusting Agent vouches or conveys this to other Agents.

The Third Party Trust Relationship has the following additional characteristics

1) Due to the $3^{\text {rd }}$ Party trust relationship, the trusting agent, who carries out the task of calculating the reputation of the trusted agent, can come to know about the repute values or opinions from the $3^{\text {rd }}$ Party Recommendation Agents. The Trusting Agent can then combine these repute values or opinions using some mathematical means to determine the reputation of the trusted agent.

During the process of combining the repute values or opinions from different $3^{\text {rd }}$ Party Agent using the mathematical means the trusting agent should take care that the repute values are of the same context and of the same time slot for which the trusting agent has to make a trusted based decision about the trusted agent.

2) The trusted agent of the $3^{\text {rd }}$ Party Trust Relationship (the trusted agent of the reputation relationship) could be Known Agents, Trustworthy Known Agents and Untrustworthy Known Agents.

3) If there is no $3^{\text {rd }}$ Party Trust Relationship then the $3^{\text {rd }}$ Party Recommendation Agent would not be able to determine the trustworthiness value / QoP / QoS of the trusted agent / product / service. 
Let us consider three logistic companies, East Field, West Field and South Field. Let us assume that East Field had interacted with West Field in the context of 'storing the goods' in December 2005 and had subsequently assigned it a trustworthiness value of ' 5 '. Further more let us assume that South Field has issued a reputation query to East Field about the trustworthiness of West Field in the context of 'Storing its Goods' and time slot of December 2005. On receipt of the reputation query East Field communicates the trustworthiness value that it had assigned for West Field in context of 'Storing the goods' and time slot of December 2005. As we can see from above that the trustworthiness value assigned by East Field to West Field becomes the repute value of West Field when East Field communicates the trustworthiness value to other agents.

\section{Third Party Trust Relationships}

In this section, we will formally define and elucidate the Reputation Query Trust Relationship. Additionally we enumerate the difference between the Reputation Query Relationship and the Trust Relationship.

Definition: The Reputation Query Trust Relationship is defined as the bond or association between the trusting agent and the $3^{\text {rd }}$ Party Recommendation Agent in the reputation relationship that signifies request for recommendation by the Trusting Agent to the $3^{\text {rd }}$ Party Recommendation Agent.

The reputation query trust relationship, can be regarded as an instance of a trust relationship, in which the role of trusting agent is played by the trusting agent in the reputation relationship, the role of the trusted agent is played by the $3^{\text {rd }}$ Party Recommendation Agent in the reputation relationship, the context of the trust relationship is 'soliciting recommendation' at a given time slot. We term the relationship between the trusting agent and the $3^{\text {rd }}$ Party Recommendation Agent as Reputation Query Trust Relationship because it this relationship emancipates and revolves around the issuing of the reputation query by the trusting agent to the $3^{\text {rd }}$ Party Recommendation Agent.

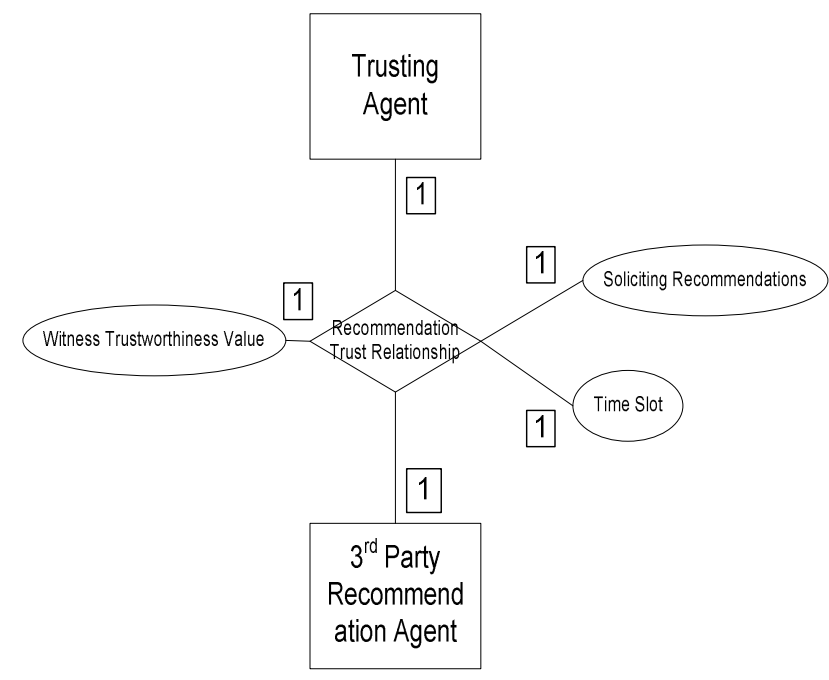

Figure 3. The Reputation Query Trust Relationship Diagram

The layout of the Reputation Query Trust on Relationship Diagram above (Figure 3) is similar to the Trust Diagram introduced in [1]. However, the role and correspondingly the name of each entity involved in the Reputation Query Trust Relationship diagram is different from role and the names of the agents involved in the Trust Relationship Diagram, except for the "Trusting Agent". The reputation query trust relationship generates the witness trustworthiness value for the Recommendation Agent or $3^{\text {rd }}$ Party Agent or Witness Agent.

There is a 1:1 (One-to-One) relationship between the Trusting Agent and a given Third Party Recommendation Agent in a Reputation Query Trust Relationship. This means that in a given Reputation Query Trust Relationship captures the relationship that a given Trusting Agent has with a single $3^{\text {rd }}$ Party Recommendation Agent, in the context of soliciting recommendations and at a single time slot. If a given Trusting Agent has relationships with more than one $3^{\text {rd }}$ Party Recommendation Agent, in the context of soliciting recommendations, then each of the relationships (that the $3^{\text {rd }}$ Party Recommendation Agent has) with the trusting agent is regarded as a distinct Reputation Query Trust Relationship. Similarly, if a given Trusting Agent has relationships with more a single $3^{\text {rd }}$ Party Recommendation Agent, in the context of soliciting recommendations, in more than one time slot, then each of the relationships (that the Trusting Agent has) with the $3^{\text {rd }}$ Party Recommendation Agent in a distinct time slot is regarded as a distinct Reputation Query Trust Relationship.

The strength of the reputation query trust relationship depicts whether the trustworthiness of the Third Party 
recommendation Agent in the context of soliciting recommendations.

\subsection{The difference between Trust and Reputation Query Trust Relationship}

Despite the similarity of the above diagram to the Trust Model described in [1], there are fundamental differences between the Recommendation Trust Relationship diagram and the Trust Model. These are:

1) As mentioned before, a Reputation Query Trust Relationship can a be regarded as a trust relationship between the Trusting Agent and a $3^{\text {rd }}$ Party Recommendation Agent in the context of soliciting recommendations ONLY at a given point in time. The role of the trusting agent is played by the trusting agent in the reputation relationship. The role of the trusted agent is played by the $3^{\text {rd }}$ Party Trust Relationship in the reputation relationship.

2) The Trustworthiness Value of the Recommendation Trust Relationship (Witness Trustworthiness Value) only represents the willingness and capability of the $3^{\text {rd }}$ Party Agent in communicating correct recommendations, at a given point in time.

However the trustworthiness of the Trust Relationship represents the willingness and capability of the trusted agent in a given context and at a given point in time.

Therefore, the Reputation Query Trust Relationship is not the same as the trust relationship. It can be regarded as an instance of the trust relationship.

The Reputation Query Trust Relationship has the following characteristics:

1) Due to the reputation query trust relationship, the $3^{\text {rd }}$ Party Recommendation Agent can come to know about the request for reputation by the trusting agent.

2) The trusted agent of the Reputation Query Trust Relationship (the trusted agent of the reputation relationship) could be Known Agents, Unknown Agents, Trustworthy Known Agents and Untrustworthy Known Agents.

3) A trusting agent may have reputation query trust relationship with more than one $3^{\text {rd }}$ Party Recommendation Agents, who are willing to give their opinions.

4) Additionally a given $3^{\text {rd }}$ Party Recommendation Agent can reply to zero-to-many (0: $\mathrm{M}$ ) reputation queries

5) If there is no reputation query trust relationship, then request for reputation could not reach the $3^{\text {rd }}$ Party Recommendation Agent and subsequently the reputation value for the Trusted Agent could not be generated.

\section{Conclusion}

In this paper, we proposed a definition for reputation relationship.From the analysis of reputation relationship we proposed that the reputation relationship is a composite relationship and is composed of three inner relationships.

Furthermore, we defined the Reputation Query Trust Relationship, Third Party Trust Relationship and the Recommendation Trust Relationship. Furthermore, we explained the difference between each of the inner reputation relationships and Trust Relationship.

\section{References}

[1]Chang, E., Dillon, T.S., Hussain, F.K., 'Trust and Reputation for Service Oriented Environment', John Wiley and Sons (To be published in 2005). 